\title{
LE BRÉSIL, PAYS D'IMMIGRATION
}

\author{
Pat Maurice-Ed. Perret ${ }^{1}$
}

Avec 2 figures

\section{Introduction}

Pourquoi le Brésil, malgré son étendue, n'a-t-il guère plus de quarante millions d'habitants ?2 Ses $8511189 \mathrm{~km}^{2}$ le mettent au quatrième rang des états du monde; il est plus grand que les Etats-Unis, presque autant que l'Europe entière. Cette disproportion étonnante a déjà attiré l'attention de géographes, en particulier de F. FrEISE et de P. James. Le Brésil n'est pas stérile ou désertique, on admet généralement que les quatre cinquièmes de sa superficie sont productifs; bien que la plus grande partie du pays soit située dans la zone tropicale, par conséquent n'ait pas un climat très favorable aux populations blanches, de vastes régions sont en dehors des tropiques et jouissent d'un climat tempéré; les ressources naturelles sont nombreuses: grandes forêts, minéraux, pierres précieuses, sol fertile. A. PENCK estime que le Brésil peut nourrir plus d'un milliard d'habitants. Frerse qui a étudié la question de près juge, en 1936, ce chiffre exagéré. Selon lui, le Brésil n'a de place que pour 450 à 550 millions d'habitants, mais il he considère qu'un pays essentiellement agricole, il envisage à peine le fait que des méthodes techniques perfectionnées permettront un meilleur rendement des ressources naturelles et surtout il pense que l'industrie ne jouera jamais qu'un rôle très secondaire. Ses conclusions sont en partie démenties par les tendances économiques actuelles du Brésil et il est probable que ce territoire a une capacité d'absorption plus grande que ce que FREISE prévoyait.

A nos yeux, les causes de la faible densité de population résident dans des obstacles physiques d'une part, dans des obstacles historiques d'autre part.

\section{Obstacles physiques}

La structure du pays est simple dans ses grandes lignes, d'une part une vaste masse continentale qui s'étend des Guyanes au Rio Grande do Sul et qui n'est coupée que par la vallée de l'Amazone, d'autre part des plaines: la plaine de l'Amazonè et celle du Paraguay qui lui fait suite, les plaines côtières. Le massif continental se compose d'un socle cristallin recouvert de schistes. et quartżites plissés que surmontent des platesformes de grès. La morphologie du pays n'a pas encore été étudiée dáns tous ses détails, mais il semble qu'il n'y ait pas eu de plissement important depuis le Dévonien. Une grande étendue de ce massif a été pénéplanée, aussi on parle couramment de Plateau Brésilien, au Sud de l'Amazone et de Plateau des Guyanes, au Nord de ce fleuve. Dans ces deux plateaux, l'érosion a creusé de nouvelles vallées et modelé des croupes; ici et là subsistent des chaînes de montagnes, vestiges des plissements anciens, mais dont les plus hauts sommets n'atteignent pas 3000 mètres (Pico da Bandeira, dans la Serra do Caparaó, état d'Espirito Santo, 2890 mètres; Mont Roraima, à la ftontière du Brésil, du Vénézuela et de la Guyane anglaise, 2875 mètres; Agulhas Negras, dans la Serra do Itatiaia, état de Rio de Janeiro, 2787 mètres $^{3}$ ). Le Plateau Brésilien est incliné doucement vers l'intérieur; du côté de l'Atlantique, il se termine brusquement par des escarpements raides et élevés, en avant desquels se trouvent des contreforts et des montagnes isolées. La plupart des rivières qui prennent naissance sut le plateau descendent la pente vers

$$
\text { a Berne. }
$$

1 Etude basée sur une conférence donnée le 26 janvier 1946 \l'Union' Suisse, Société d'Etudes pour la Colonisation d'outré-mes,

Recensement de 1940: 41236779 babitants. Eväluation aul ler janvier 1944: 44400000 habitants.

a Chiffres tirés de la demiére édition du livre «Brasil» (1944). Les alittudes varicnt selon les livres; selon certains les Agulhas N'cgras semient le plus haut sommet du pays. 
l'intérieur, soit du côté de l'Amazone, soit du côté du Paraguay; presque toutes forment des chutes ou des rapides qui empêchent la navigation; dans tous les états, de nombreuses localités portent le nom de cachoeira, cachoeiro (portugais: rapides ou chute), ou leurs diminutifs cachoeirinha, cachoeirinho, soit comme noms simples, soit en formes composées, Bom Jesus da Cachoeira Alegre, Cachoeiro do Itapemirim, ou bien de salto (saut), Salto Grande do Paranapanema, Sao Sebastiao do Salto Grande, ou encore de itú (tupy: y eau et tú chute), Ituassú (grande chute d'eau), Ituverava (chute d'eau qui brille). Ces appellations sont parmi les noms de lieu descriptifs les plus fréquents. La côte est très peu découpée; sa longueur, au Brésil n'est que d'environ 7900 kilomètres, soit moins de la moitié des côtes de Norvège; elle offre cependant de bons ports naturels, mais les plaines côtières n'occupent nulle part une grande étendue; en beaucoup d'endroits, elles sont coupées par des marais ou des lagunes, foyers de malaria et de fièvre jaune, et l'intérieur est difficile d'accès car les escarpements bordant le plateau vers la côte sont recouverts d'une forêt très épaisse et difficilement pénétrable. Le seul grand fleuve navigable sur un long parcours et jusqu'à son embouchure, l'Amazone, est, sur territoire brésilien, presque sans interruption bordé de forêts tropicales très denses dont beaucoup sont périodiquement inondées.

On voit ainsi que le Brésil offre, au premier abord, un aspect peu propice à la colonisation et même à l'exploration. Ce n'est que tout récemment que les régions marquées "terra incognita», ou les rivières indiquées en pointillé, au centre du pays, ont disparu des cartes géographiques.

\section{Obstacles historiques}

Le Brésil, avant même d'être découvert, appartenait déjà au Portugal. En effet, en 1494, les rois de Portugal et d'Espagne signèrent le traité de Tordesilhas par lequel une ligne située à peu près sur le $49^{\circ}$ longitude Ouest diviserait les terres à découvrir entre les Espagnols et les Portugais, les premiers obtenant les régions occidentales, les autres, les régions orientales. C'est ainsi qu'après la découverte du Brésil en 1500 par PEDRO Alvares Cabral, le pays fut occupé par les Portugais et resta dès lors la seule contrée de langue portugaise de l'Amérique.

Les Portugais fondèrent très vite quelques établissements le long de la côte: Sao Salvador ${ }^{4}$, en 1502, colonie qui au début ne fut pas prospère, Sao Vicente, en 1532, Olinda, en 1537. Ne trouvant pas au Brésil de richesses à portée de la main, ils durent se résoudre à cultiver le sol, fait nouveau dans l'histoire de la colonisation. En effet, jusqu'alors, les Européens qui s'étaient rendus dans des pays d'outre-mer s'étaient bornés à l'acquisition ou à l'exploitation de matières premières - bois, épices, métaux précieux - ou à des échanges divers avec les indigènes. Les nouveaux maîtres du pays, ayant quelque difficulté à s'adapter au climat et ne réussissant pas à faire travailler les Indiens pour eux, durent user de l'esclavage et dès 1538 des nègres furent amenés d'Afrique au Brésil; la traite des noirs continua pendant plus de trois siècles et ce n'est qu'en 1888 que l'esclavage fut officiellemeht aboli.

Les colons portugais, bien qu'ils ne fussent pendant longtemps pas très nombreux, furent très jaloux de conserver leur souveraineté au Brésil. A plusieurs reprises, des étrangers, Français, Hollandais, Anglais, établirent des colonies sur la côte, mais celles-ci furent toutes éphémères. Tant que le Brésil dépendît du Portugal, l'immigration étrangère fut interdite; les propriétaires fonciers trouvaient suffisamment de main d'œuvre parmi les esclaves, le commerce n'existait qu'avec la mère patrie - les ports n'étaient ouverts qu'aux seuls navires portugais - et l'industrie toujours vue d'un mauvais œil depuis Lisbonne fut, par décret daté de 1785, complètement interdite, tant le Portugal craignait que la colonie ne devînt trop puissante et ne secouât le joug royal.

\footnotetext{
- Sao Salvador est connu généralement sous le nom de Bahia qui, au Brésil, désigne l'état dont Sao Salvador cst la capitalc.
} 
Les maitres portugais admirent, occasionnellement, quelques étrangers isolés, mais à une condition absolue, c'était qu'ils fussent catholiques ou se convertissent. Le Brésil ne fut pas, comme les Etats-Unis, une terre d'élection pour les non-catholiques persécutés pour leur religion.

Jusqu'au XIXe siècle, la colonisation de l'intérieur du pays progressa lentement. En 1554 déjà, des Jésuites, utilisant le point le plus accessible de l'escarpement du côté de l'Atlantique, fondèrent une mission à Sao Paulo, à 1100 mètres d'altitude, mais pendant longtemps cet établissement eut peu d'importance. La découverte, dès 1698, de mines d'or, puis de mines de diamant, au centre de l'état de Minas Gerais (les mines générales), fut l'origine d'un peuplement relativement important de blancs et d'esclaves travaillant dans les mines. On comptait environ 80000 personnes dans les régions minières, au milieu du XVIIIe siècle. Les autres régions de l'intérieur ne connurent pas de période brillante comme Minas Gerais. Lentement et graduellement, la colonisation s'avança de la côte vers l'intérieur, dirigée seulement par des Portugais, comme l'attestent les noms de lieu de tout le pays. Le Brésil n'était pas, comme les Etats-Unis, un pays ouvert de divers côtés; il ne vit rien de comparable aux expéditions espagnoles qui, du Mexique, conquirent, d'un côté la Californie, de l'autre le Texas et le Nouveau Mexique, ni aux expéditions françaises qui, du Canada, pénétrèrent dans la région des lacs, descendirent la vallée du Mississipi, fondèrent la colonie de la Louisiane.

Les Portugais restèrent seuls maîtres et comme leur patrie n'était pas un réservoir d'hommes aussi important que d'autres états d'Europe, la population du Brésil ne put augmenter rapidement. Vers 1800, on l'estimait seulement à 2630000 habitants, soit 430000 blancs, 1500000 nègres et 700000 Indiens, alors que les Etats-Unis, au recensement de 1800, en comptaient déjà 5308483.

\section{L'immigration moderne}

En 1808, le roi de Portugal et la cour qui, menacés par les troupes de Bonaparte avaient dû émigrer, s'établirent au Brésil. Un des premiers actes du monarque fut d'ouvrir les ports à la navigation internationale et d'autoriser les industries. Il décida ensuite de laisser entrer les colons étrangers. Cédant aux instances de SÉBASTIEN Nicolas GACHET, agent du canton de Fribourg, plus tard nommé consul de Suisse, il fit venir cent familles de ce canton. Ces Suisses, au nombre de 1085, s'embarquèrent en 1819 et l'année suivante fondèrent la colonie de Nova Friburgo, dans les montagnes, au Nord de Rio de Janeiro. Cette colonie, mal organisée, passa par de grandes difficultés, mais un petit noyau subsista et aujourd'hui, dans la petite ville qui est maintenant une localité prospère, on trouve encore quelques familles aux noms fribourgeois.

Après la proclamation de l'indépendance du Brésil, en 1822, l'empereur résolut de développer le pays et favorisa l'immigration. Pour renforcer la frontière du Sud, il fit venir des colons allemands qui, dès 1829, s'établirent dans le Rio Grande do Sul. En 1843 , le gouvernement décida de supporter les frais de voyage des immigrants et de les aider à s'établir. Mais il ne suffisait pas de créer des colonies, il fallait qu'elles puissent vivre et les difficultés — éloignement des grandes villes, manque d'écoulement des produits agricoles, mauvais moyens de transports - entravèrent leur développement. En 1859, sur les plaintes des colons allemands établis au Brésil, l'Allemagne interdit toute émigration vers ce pays (décret von HEYDT). La situation n'était pas aussi sombre que les colons l'avaient décrite, car la population des colonies allemandes du Rio Grande do Sul qui, au moment du décret von Herdr, était de 20493 habitants, se multiplia tant que cinquante ans plus tard, on estimait à plus de 200000 le nombre des Rio Grandais de race allemande issus du premier groupe.

En 1867 fut établie la ligne de chemins de fer de Santos à Sao Paulo. Cette construction coïncide avec le début du développement de l'état de Sao Paulo. Vers 1870, on 


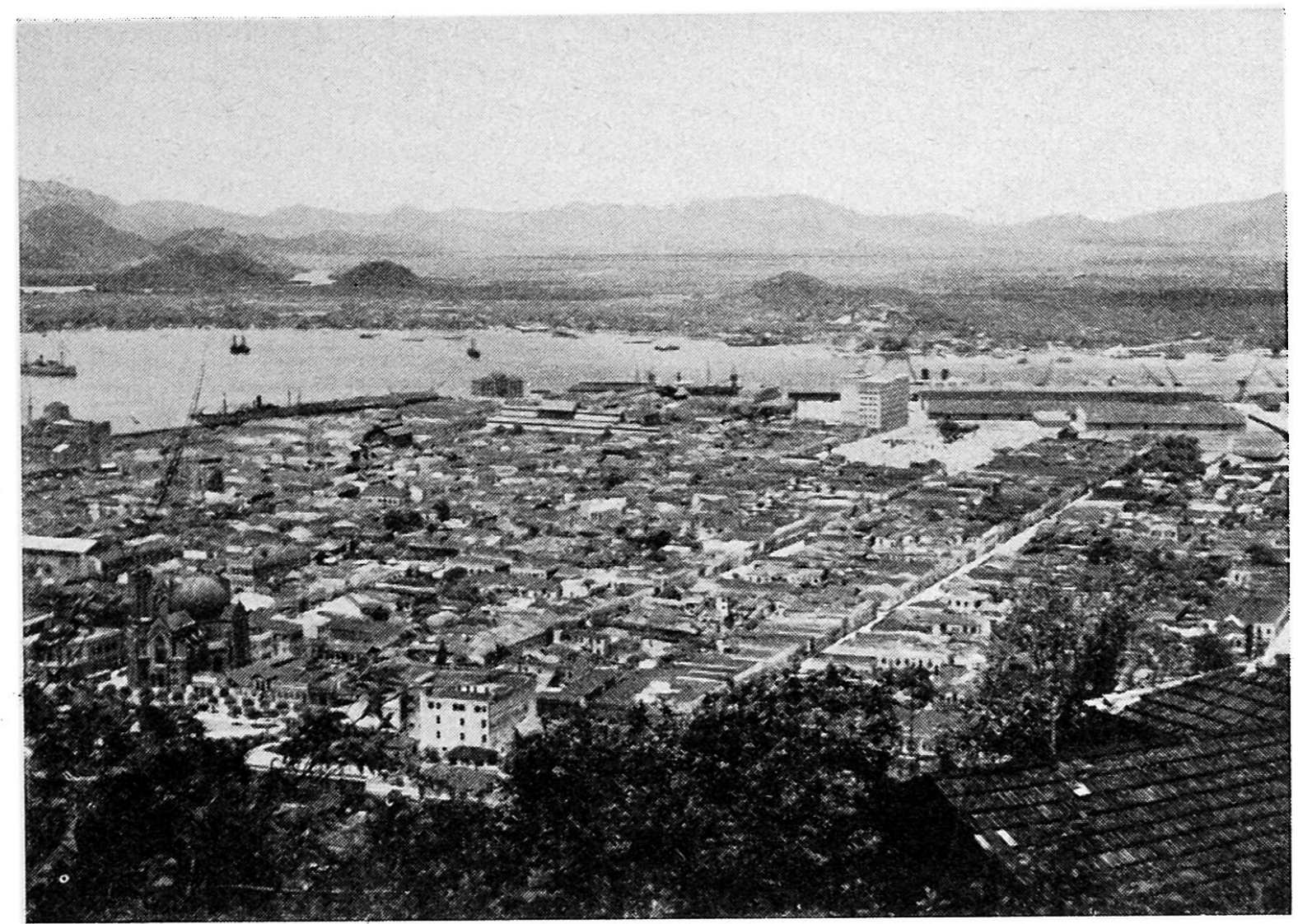

Fig. 1. Santos, la plaine còtiere, à l'arrière-plan les contrcforts de la « Serra ». Photo M. E. Perret

s'aperçut que le sol de vastes régions, la «terra roxa» - terre violette, sol éluvial des diabases - convenait admirablement à la culture du café, mais il n'y avait pas de main d'œuvre et il était difficile de se procurer des esclaves, car la traite des noirs avait été interdite depuis 1830. On décida alors de faire appel à des immigrants européens. En 1871 et 1872, 355000 personnes furent ainsi engagées, en très forte majorité des Italiens (parmi lesquels pas moins de 100000 Piémontais). L'abolition de l'esclavage et la chute de l'empire qui la suivit de près créèrent un marasme qui fut senti surtout dans les régions de vieille colonisation, les grandes plantations des régions de Pernambouc, de Bahia, de Rio de Janeiro, de Minas Gerais, où les nègres libérés se refusèrent en grand nombre à continuer à travailler pour leurs anciens maîtres. Sao Paulo profita de la situation, devint le premier état producteur de café et prit une place prépondérante dans le pays. L'immigration, qui avait baissé pendant quelques années, reprit de plus belle, elle se dirigea presque exclusivement sur Sao Paulo qui, en 1901, absorbait le 84 pour-cent des immigrants arrivés au Brésil. Comme on ne pouvait obtenir suffisamment de colons européens, le Brésil engagea des négociations avec le Japon. Les premiers immigrants de ce pays, au nombre de 781, arrivèrent en 1908; chaque année de nouveaux groupes les suivirent, certaines années très nombreux (1929: 16648; 1933: 24494), puis, dès 1935 , pour des raisons politiques, l'immigration japonaise diminua fortement et cessa complètement en 1941.

En 1921, pour la première fois, le gouvernement se décida à réglementer l'entrée dans le pays. Puis, en 1930, à la suite du chômage régnant dans le pays, l'immigration fut restreinte aux seules personnes en possession d'une «lettre d'appel» (carta de chamada), émise au Brésil, soit par le gouvernement d'un des états, soit par des parents, soit par des sociétés agricoles ou des propriétaires agricoles individuels. En 1936, à l'instar des Etats-Unis, on établit un système de contingentement, chaque pays étant 
autorisé à envoyer annuellement un nombre d'émigrants à destination du Brésil, représentant le deux pour-cent du nombre total des personnes de cette nationalité fixées au Brésil au cours des cinquante dernières années. Ce contingent est actuellement de 3000 pour la Suisse. ${ }^{5}$ On vient de reconnaître la nécessité d'une nouvelle immigration et des lois promulguées récemment vont offrir de grandes facilités aux nouveaux colons, tout spécialement aux agriculteurs.

De 1884 à 1943, alors que 30766000 immigrants arrivaient aux Etats-Unis, 4022975 immigrants entrèrent au Brésil, se répartissant comme suit:

$\begin{array}{lrlllr}\text { Portugais } & 1224141 & \text { Autrichiens } & 85836 & \text { Lithuaniens } & 28602 \\ \text { Italiens } & 1142128 & \text { Turcs } & 78490 & \text { Anglais } & 25275 \\ \text { Espagnols } & 582793 & \text { Polonais } & 48673 & \text { Argentins } & 23930 \\ \text { Japonais } & 188615 & \text { Roumains } & 39261 & \text { Yougoslaves } & 22907 \\ \text { Russes } & 184174 & \text { Français } & 33058 & \text { Suisses } & 10758 \\ \text { Allemands } & 172326 & \text { Syriens } & 29570 & \text { Divers } & 102438\end{array}$

Le Brésil est parmi les pays où les Suisses émigrent volontiers, comme le montrent les statistiques. ${ }^{6}$

Les noms de lieux ne donnent qu'un reflet très atténué de l'immigration étrangère. Le Brésil n'offre pas la bigarrure des Etats-Unis où les noms anglo-saxons voisinent avec les noms indiens, espagnols, français, hollandais, allemands, grecs, latins. Les Portugais ont souvent adopté les noms indiens (principalement tupys) qu'ils trouvaient et partout ailleurs ont donné des noms descriptifs, des noms religieux ou des noms de lieux de leur patrie. Même dans les états où l'immigration de groupes étrangers a été nombreuse - Sao Paulo, Rio Grande do Sul, Parana, Santa Catarina - les noms rappelant le pays d'origine des colons ne désignent, à l'exception de la ville de Blumenau qui a pris le nom de son fondateur, un chirurgien allemand, que de petites localités ou des colonies, par exemple: Grunejaeger, Hamburger-Berg, Karlsruhe, Lindenau, - Marienthal, Neu-Breslau, Nova Bremen, Nova Danzig, Nova Wurtemberg, Novo Hamburgo; Garibaldi, Monte Veneto, Nova Belluno, Nova Bergamo, Nova Milao, Nova Padua, Nova Palermo, Nova Roma, Nova Sardenha, Nova Trento, Nova Tyrol, Nova Treviso, Nova Veneza, Nova Vincenza, ou encore Helvetia, Nova Odessa, Nova Polonia. Quelques-uns des noms religieux donnés à des localités dans les états du Sud perpétuent des saints ou saintes qui sont vénérés surtout en dehors du Portugal: Santa Adelia, Santa Coleta, Santa Tecla, Sao Jacob, Sao Leopoldo, Sao Nicolau, Sao Vendelino, Sao Xavier. Le nombre des noms à consonance étrangère augmente, mais ce sont généralement les autorités brésiliennes ou les compagnies de chemins de fer qui nomment des localités ou des stations de chemins de fer en mémoire de personnages marquants, au nombre desquels se trouvent des hommes d'origine germanique, italienne, slave, française ou anglo-saxonne, tels Felippe Schmidt, Machinista Mezzati, Engenheiro (ingénieur) Trompowski, Marechal Mallet, Engenheiro Dunham. Comme dans quelques cas le fait s'est déjà produit, il est probable qu'avec le temps on ne gardera que la seconde partie du nom; puis, pour suivre les règles de la nouvelle orthographe, on écrira tous ces noms phonétiquement et ils perdront alors tout caractère étranger.

\section{L'immigration future}

Comme nous l'avons dit au début de cet article, la majeure partie du Brésil est située sous les tropiques et le climat en est peu favorable à la colonisation européenne. Toute la plaine de l'Amazone a, selon l'échelle de W. KoEPPEN, un climat Af (climat tropical

\footnotetext{
- Information de M. Pires do Rio, chargé d'affaires du Brésil en Suisse.

- Nombre d'émigrants suisses partant pour le Brésil: 1924192819321939 Nombres absolus . . . . . . . . . . . . . . . . . . . . . . . . . . . . . . . $317 \quad 212 \quad 812$

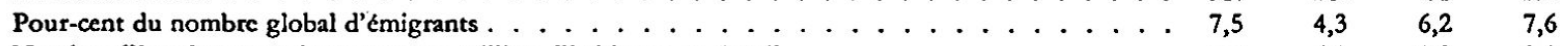
Nombre d'immigrants suisses pour un million d'habitants au Brésil . . . . . . . . . . . . . . . . . . . $\quad .7,8 \quad 5,3 \quad 2,0 \quad 3,8$
} 


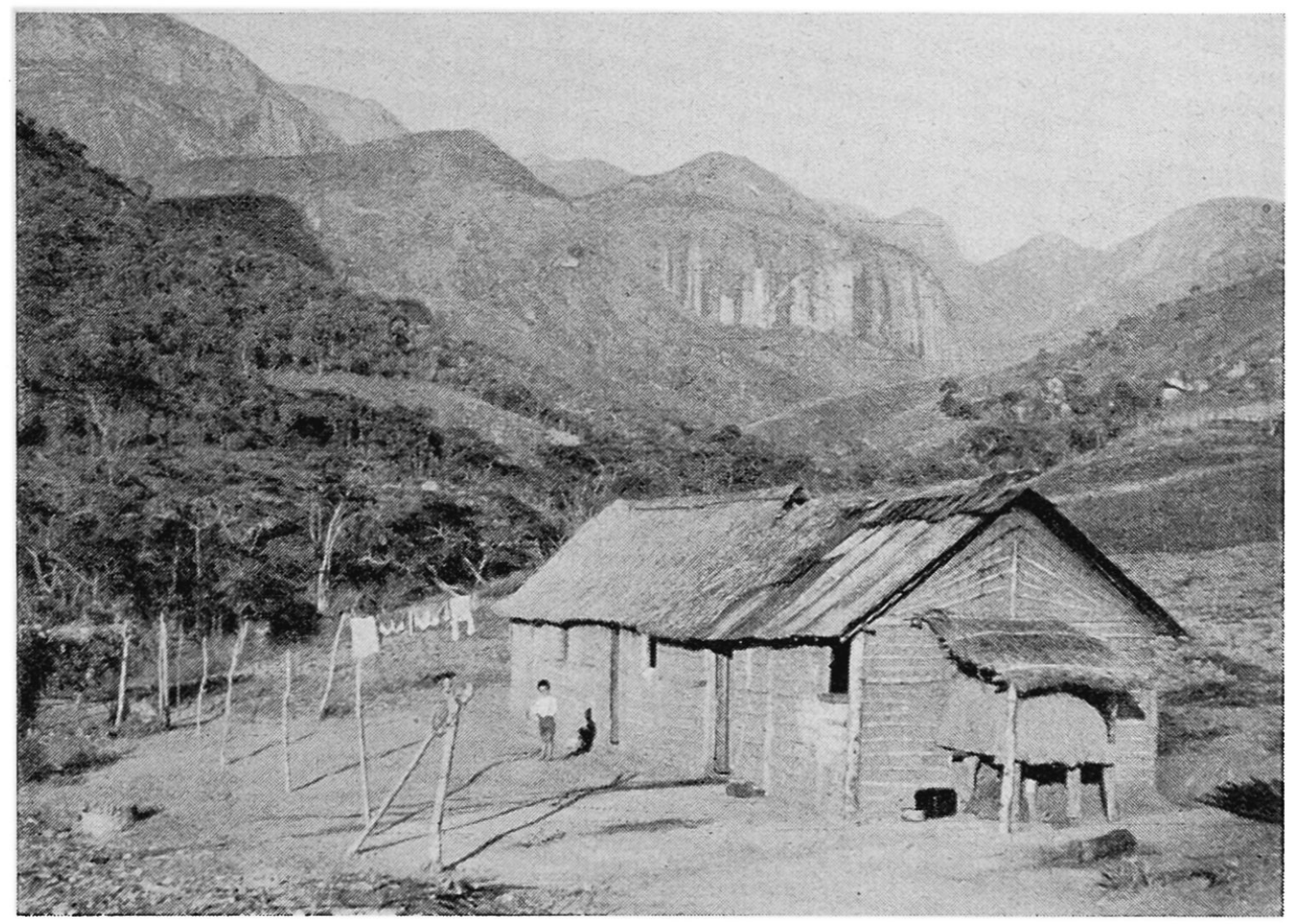

Fig. 2. Une haute valléc dans la «Serra dos Orgaos, bord du Plateau Brésilien, au Nord de Rio de Janciro. Photo M. E. Perret

avec pluies distribuées toute l'année); le Plateau des Guyanes et la majeure partie du Plateau Brésilien sous les tropiques sont de climat Aw (climat tropical avec pluies d'été); les plaines côtières, des Guyanes jusqu'à Santos, ont un climat tropical également (Af ou Aw). Mais il reste de vastes territoires de climats tempérés propres à une colonisation intense. Ceux-ci couvrent une partie des états de Rio de Janeiro, Mato Grosso et Goíaz, la plus grande partie des états de Minas Gerais et Sao Paulo, la totalité des états de Parana, Santa Catarina et Rio Grande do Sul. Ils sont, soit de type Cwa (étés chauds, pluies d'été), dans la partie Nord, soit de type Cfa (étés chauds, pluies distribuées pendant toute l'année), au Sud. Il y a quelques ilôts de type Cwb ou Cfb (étés tempérés), dans les régions les plus élevées. A titre de comparaison, notons que le climat Cfa est celui de la plaine du Pô, de la plaine hongroise et de tout l'Est des Etats-Unis, au Sud du $40^{\circ}$ latitude et jusqu'aux Montagnes Rocheuses. E. de MARTONNE, lui, assimile le climat des états du Sud du Brésil au climat chinois (climat de mousson), ce qui le rapproche du climat de la plus grande partie de la Chine, du SudEst des Etats-Unis, du Natal et de la côte de la Nouvelle-Galles du Sud. Selon l'une ou l'autre classification, ce sont des climats très propices aux populations européennes. Le Brésil, dans son ensemble étant situé à l'Est d'un continent, est, par suite de la rotation de la terre et des zones de haute pression sur l'Atlantique Sud, soumis aux influences des vents venant de la mer, vents qui ne sont nulle part arrêtés par de hautes chaînes de montagnes. Les différences de température entre l'été et l'hiver ne sont pas accentuées, l'humidité est, en général, relativement élevée ainsi que la moyenne annuelle des précipitations. ${ }^{7}$

\footnotetext{
7 Moyenne annuclle des précipitations dans le Sud: Sao Paulo, $142 \mathrm{~cm}$., Porto Alegrc, $122 \mathrm{~cm}$. (Zurich, $103 \mathrm{~cm}$.); températures moyennes: Sao Paulo: $21,4^{\circ} \mathrm{C}$. en janvier, $15,2^{\circ} \mathrm{C}$. en juillet; Porto Alegre: $24,9^{\circ} \mathrm{C}$. en janvier, $13,9^{\circ} \mathrm{C}$. en juillet (Zurich: $-1,4^{\circ} \mathrm{C}$. en ianvier, $18,4^{\circ} \mathrm{C}$. cn juillet).
} 
Bien que la plus grande partie des meilleurs sols - en particulier la terre violette et les sols basaltiques sur les bords du Plateau Brésilien dans le Rio Grande do Sul et à Santa Catarina - soient déjà défrichés, il reste encore de vastes étendues couvertes de forêts abondantes dont le sol est très fertile. Les nouvelles colonies devront suivre la trace des anciennes. Le défrichement de la forêt sera le premier travail agricole, car si, dans la forêt qui couvre la plus grande partie du pays, il y a bien des clairières qu'il serait facile de cultiver directement, celles-ci ont un sol pauvre qui ne donnera jamais de récoltes abondantes.

Les obstacles physiques à l'immigration ont été en grande partie vaincus et même aujourd'hui certains d'entre eux constituent des sources de richesse: les nombreuses chutes d'eau commencent à être exploitées et fourniront une source quasi inépuisable d'énergie hydro-électrique, les forêts offrent divers produits de valeur (bois, maté, caoutchouc, etc.). Certains marais ont été asséchés donnant ainsi à l'agriculture de nouveaux terrains bien situés et réduisant les risques de maladies. Des chemins de fer conduisent maintenant des ports vers l'intérieur et relient les principales villes. Le gouvernement de GÉtulio VARGAS avait fait le plan de doter le pays d'un réseau de grandes routes d'une longueur totale de 35778 kilomètres et il est à espérer que le gouvernement actuel mettra ce programme à exécution. Enfin les lignes d'aviation se sont beaucoup développées et jouent un grand rôle dans la vie économique. En 1943, leur réseau avait une extension de 91351 kilomètres et les avions avaient transporté 171860 passagers, 3000 tonnes de bagages, une même quantité de marchandises, $557000 \mathrm{~kg}$. d'envois postaux.

Le Brésil, il y a encore quelques années, était un pays presque exclusivement agricole et producteur de matières premières. Encore actuellement, l'agriculture est à la base de l'économie du pays. Quatre produits sont de beaucoup les plus importants, ce sont le maïs, dont les cultures couvrent une superficie de $4266211 \mathrm{ha}^{8}{ }^{8}$, le café $(2490855$ ha.), le coton (2413562 ha.) et le riz (1171755 ha.) Le maïs et le riz sont à la base de l'alimentation brésilienne; le café est encore le principal produit d'exportation. Mais récemment l'industrie s'est beaucoup développée, surtout depuis la guerre qui a presque complètement tari les sources de produits manufacturés d'Europe et des Etats-Unis. Les industries en 1942 occupaient 847000 ouvriers, principalement dans l'état de Sao Paulo, à Rio de Janeiro, dans les états de Pernambouc, Minas Gerais et Rio Grande do Sul. L'industrie textile vient en premier lieu et le Brésil depuis la guerre s'est même mis à exporter des quantités importantes de tissus de coton dans les autres pays d'Amérique du Sud. La métallurgie s'est aussi développée, mais lentement, car quoique les mines de fer soient extrêmement riches, elles n'ont pu encore donner lieu à une industrie lourde semblable à celle de la Ruhr, du Nord-Ouest anglais, de Pittsburg ou de Duluth aux Etats-Unis, par suite du manque de houille sur place et de la difficulté d'en amener. En effet, les mines de fer sont dans l'état de Minas Gerais, loin de la côte et les seuls gisements importants de houille au Brésil sont situés dans les états de Santa Catarina et de Rio Grande do Sul. Mais ce n'est plus pour longtemps que le Brésil restera en arrière, car depuis 1942, on est en train de travailler activement à la construction d'un centre sidérurgique brésilien à Volta Redonda, entre Rio de Janeiro et Sao Paulo et entre les mines et la côte. 112 ingénieurs, 110 dessinateurs, 17000 employés et ouvriers ont coopéré pour mener à bien ce projet. Plusieurs des usines sont déjà en activité et ce centre qui utilisera les minerais de Minas Gerais et le charbon de Santa Catarina est appelé à jouer un grand rôle dans la métallurgie mondiale. C'est un exemple remarquable de plan d'aménagement national.

En conclusion, nous constatons que le Brésil est un pays en plein développement et qui offre beaucoup d'avenir, mais il doit augmenter sa

B Statistiques de 1943. 
population par l'immigration pour pouvoir tirer le meilleur parti de ses richesses.

Les colons européens se dirigeront de préférence vers les états du Sud. Ceux-ci ont encore une très grande capacité d'absorption. Sao Paulo dont la superficie est six fois celle de la Suisse n'a pas encore huit millions d'habitants; il y en a encore moins dans les autres états, Minas (plus grand que la France), Parana (égal à la moitié de l'Italie), Santa Catarina (deux fois la Suisse), Rio Grande do Sul (aussi grand que l'Italie). ${ }^{9}$ Quant aux régions tropicales, elles se peupleront par des migrations internes, car les personnes nées au Brésil, même dans le Sud, sont plus aptes à en supporter le climat.

Principales sources utilisées:

1. Blumenauer Volkskalender für das Jahr 1935. Blumenau 1934.

2. Carta Geographica do Brasil organisada pelo Club de Engenharia do Rio de Janeiro, 1:275000. 1922.

3. DENIS, P.: Le Rrésil au XXe siècle. Paris 1909.

- Amérique du Sud I. Paris 1927.

4. Diccionario Chorographico Brasileiro. Rio de Jareiro 1929.

5. FREISE, F.: Brasiliens Bevölkerungskapazität. Petermanns Mitteilungen, 82, 1936, S. $143-147$.

6. FREYRE, G.: Casa Grande e Senzala. Rio de Janeiro 1936.

7. GUTERSOHN, H.: Sao Paulo. Vierteljahresschrift der Naturforschenden Gesellschaft Zürich, 85, 1940, S. 149-255.

- Das Kemland von Minas Gerais. Arbeiten Geographisches Institut Eidgenössische Technische Hochschule Zürich. Nr. $3,1945$.

8. JAMES, P.: Latin America. New York 1942.

9. LUFFT, H.: Lateinamerika. Leipzig 1930.

10. MAURETTE, F., ec SIEVERS, E.: L'Immigration et la Colonisation au Brésil. en Argentine et en Uruguay, I, Brésil. Revue Internationale du Travail, 35, 1937, p. 230-263.

11. METTLER, A. : Les factcurs géographiques dans la découverte et l'occupation du Brésil. Annales de Géographie, 46, 1937, p. 61-75.

12. Ministerio das Relaçoes Exteriores: Brasil 1940-1944. Rio de Janeiro.

13. PAUWELS, P.: Aclas Geographico Geral e especialmente do Brasil, Sao Paulo, Cayciras. Rio de Janeiro 1936.

11. PENCK, A.: Das Hauptproblem der physischen Anthropogeographie. Sitzungsberatungen der Preussischen Akademie der Wissenschaften 22, 1924, S. 242-257.

13. SCHMIEDER, O.: Länderkunde Südamerikas. Leipzig und Wien 1932.

Plusieurs de ces ouvrages en particulier les numéros $3,7,8,15$, contiennent des bibliographies détaillées.

- Selon des évaluations au 1 janvier 1944, la population de ces états était la suivante: Sao Paulo, 7733500; Minas Gerais, 7310000; Parana, 1279700; Santa Catarina, 1255900; Rio Grande do Sul, 3578600.

\title{
ZENTRALINDISCHE FRUCHTBARKEITSRITEN UND IHRE BEZIEHUNGEN ZUR INDUSKULTUR
}

\author{
Von Wilhelm Koppers
}

Mit 8 Abbildungen

\section{Einleitung}

$\mathrm{Da}$ der Aufdeckung der Induskultur für weite Bereiche der Indienforschung eine umwälzende Bedeutung zukommt, wird heute von den maßgebenden Wissenschaftern allgemein anerkannt. Wenn Sir MARSHALL schon vor fünfzehn Jahren darauf hingewiesen hat, daß eine Anzahl der Wesenselemente des heutigen Hinduismus auf die Induskultur zurückgeführt werden muß, so hat die Forschung der nachfolgenden Zeit dieses Resultat nicht nur bestätigen, sondern um manche bedeutsame Punkte erweitern können ${ }^{1}$. Vom Standpunkte bestimmter Fruchtbarkeitsriten aus, die ich selbst in Zentralindien bei den Primitivstämmen der Bhil und der Gond zu beobachten Gelegenheit hatte, ergibt sich, wie ich meine, wenn nicht eine sichere, so doch eine wahrscheinliche neue Beziehung zu bestimmten, und zwar bis jetzt dunkel und ungeklärt gebliebenen Funderscheinungen der Induskultur hin. Den Fruchtbarkeitsritus, um den es

1 J. MARSHALL: Mohenjo-Daro and the Indus Civilization. I., London 1931, S. 110 f. Vgl. R. O. EHRENFELS: Motherright in India. Hyderabad (Deccan) 1941, S. $180 \mathrm{ff}$. 